\title{
Innovative Energie- und Qualitäts-Adaptierte Lenkungsstrategien für Mobile Autonome Brecher
}

\author{
Vorstellung des Dissertationsprojektes
}

\author{
Dietmar Kemper ${ }^{1}$, Siegfried Scheibner², Matthias Egger ${ }^{2}$, Hermann Almer ${ }^{2}$ und Helmut Flachberger ${ }^{1}$ \\ ${ }^{1}$ Lehrstuhl für Aufbereitung und Veredlung, Department Mineral Resources Engineering, Montanuniversität \\ Leoben, Leoben, Österreich \\ ${ }^{2}$ SBM Mineral Processing, Liezen und Oberweis, Österreich
}

Eingegangen 16. Juli 2021; angenommen 20. Juli 2021; online publiziert 10. August 2021

\begin{abstract}
Zusammenfassung: Mobile Brechanlagen haben ein starkes Wachstum durch viele Bereiche der Primär- und Sekundärrohstoffaufbereitung - beispielsweise in Steinbrüchen für Baurohstoffe, Industrieminerale und Erze (primär) und für Baureststoffe, schadstoffentfrachtete Schlacken und diverse Verbundstoffe (sekundär) vollzogen. Dabei gingen die Bemühungen der Ingenieur*innen in den vergangenen Jahren verstärkt in Richtung immer größerer Mobilanlagen zur Steigerung der erzielbaren Durchsatzraten unter Wahrung der Transportierbarkeit auf dem öffentlichen Straßennetz.
\end{abstract}

Seitens der Forschungspartner "SBM Mineral Processing" und "Lehrstuhl für Aufbereitung und Veredlung" wird erwartet, dass Innovationen in ein weitgehend autonomes Brechen durch digitale Transformation einen weiteren Wachstumsschub in den internationalen Märkten ermöglichen werden. Dazu müssen diese mobilen Brechaggregate mit intelligenter und zugleich robuster Sensorik und Aktorik sowie modernen Algorithmen ausgestattet werden, damit diese in die Lage versetzt werden können, ihren voreingestellten Betriebszustand zu halten, Abweichungen zu erkennen und darauf richtig zu reagieren, um dann wieder in den optimalen Betriebszustand zurückkehren zu können. Bei den derzeit am Markt befindlichen Aggregaten ist das "Reagieren“ überwiegend den Maschinenbetreibern vorbehalten, deren Erfahrung und Können einen maßgeblichen Einfluss auf das wirtschaftliche Betreiben mobiler Brechanlagen hat. Der kampagnenartige Einsatz und die damit einhergehenden unterschiedlich zu verarbeitenden Rohgutarten mit ihren zerkleinerungstechnischen Spezifika

\footnotetext{
Dipl.-Ing. D. Kemper ( $\square$ )

Resources Engineering,

Montanuniversität Leoben,

Max-Tendler-Straße 4

8700 Leoben, Österreich

dietmar.kemper@unileoben.ac.at
}

Lehrstuhl für Aufbereitung und Veredlung, Department Mineral wie auch der derzeitige Stand der Technik und ein oftmals ungenügendes Prozessverständnis der Maschinenbetreiber tragen zum jetzigen Zeitpunkt dazu bei, dass diese Brechaggregate sehr häufig außerhalb des optimalen Betriebsfensters für eine energie- und qualitätsoptimierte Anlagenfahrweise betrieben werden. Ein unzureichendes Produktausbringen und ein erhöhter Wartungs- und Energieaufwand sind die unerwünschten Konsequenzen.

Die Erhebung des Stands der Technik und der am Markt befindlichen Maschinen hat gezeigt, dass bereits gewisse Aspekte des automatisierten Betriebes in mobilen Brechaggregaten vorhanden sind (z. B. das Koppeln der zuzuführenden Rohgutmenge an den Lastzustand), die Möglichkeiten eines autonomen Brechens sind aber noch bei weitem nicht erreicht. Hier ist Platz für vielfältige maschinenbauliche, prozesstechnische und mechatronische Innovationen. Ziel des Projektes ist es, durch den Bau eines Prototyps mit innovativer Sensorik und Aktorik die Grenzen des Machbaren aus technologischer wie auch wirtschaftlicher Sicht für ein autonomes Brechen (durch ein selbststeuerndes und lernendes Aggregat) auszuloten bzw. neu zu definieren und wissenschaftlich zu untermauern. Unterschiedliche neue innovative Baugruppen mit Sensoren und Aktoren sowie integrierten Steuerungsalgorithmen sollen auf Einsatztauglichkeit getestet werden. Zunächst unter kontrollierten Bedingungen im Labor und am Technikum-Versuchsstand, nachfolgend in rauen Feldversuchen.

Schlüsselwörter: Mobilanlage, Prallbrecher, Brechaggregat, Autonomes Brechen, Digitale Transformation, Sensorik, Aktorik, Algorithmus 
Innovative Energy- and Quality-adapted Steering Strategies for Mobile Autonomous Crushers-Presentation of a PhD-Project

Abstract: Mobile crushing plants have undergone strong growth in many areas of primary and secondary raw material processing - for example in quarries for construction raw materials, industrial minerals and ores (primary) and for construction residues, slags and various composites (secondary). In recent years, the efforts of the engineers have been increasingly directed towards ever larger machines to increase the achievable throughput rates while ensuring transportability on public roads.

On the part of the research partners "SBM Mineral Processing" and "Chair of Mineral Processing", it is expected that innovations in largely autonomous crushing through digital transformation will enable a further growth booth in the international markets. For this purpose, these mobile crushing units must be equipped with intelligent and, at the same time, robust sensors and actuators as well as modern algorithms so that they can be enabled to maintain their present operating state, detect deviations, and react correctly to them in order to then be able to return to the optimal operating state. With the aggregates currently on the market, "reacting" is predominantly reserved for the machine operators, whose experience and skills have a decisive influence on the economic operation of mobile crushing plants. The campaign-like use and the associated different types of raw material to be processed with their specific crushing characteristics, as well as the current state of the art and the often insufficient understanding of the process by the machine operators, contribute to the fact that these crushing units are very often operated outside the optimal operating window for an energy- and qualityoptimised plant operation. Insufficient product yield and increased maintenance and energy costs are the undesirable consequences.

The survey of the state of the art and the machines on the market has shown that certain aspects of automated operation are already available in mobile crushing units (e.g. coupling the amount of raw material to be fed to the load condition), but the possibilities of autonomous crushing are still far from being achieved. There is room here for a variety of mechanical engineering, process technology and mechatronic innovations.

The aim of the project is to sound out or redefine the limits of what is feasible from a technological and economic point of view for autonomous crushing (by means of a self-controlling and learning unit) and to underpin this scientifically by building a prototype with innovative sensors and actuators. Different new innovative assemblies with sensors and actuators as well as integrated control algorithms are to be tested for their suitability for use-initially under controlled conditions in the laboratory and at the pilot plant, afterwards in rough field tests.

Keywords: Mobile plants, Impact crusher, Crushing unit, Autonomous crushing, Digital transformation, Sensors, Actuators, Algorithm

\section{Einleitung}

Die Firma SBM Mineral Processing, im Folgenden mit SBM abgekürzt, ist ein österreichisches Traditionsunternehmen mit Firmenstandorten in Oberweis und Liezen. SBM zählt im DACH-Raum zu den führenden Komplettanbietern von Aufbereitungs- und Förderanlagen für die Rohstoff- und Recyclingindustrie und von Betonmischanlagen für Transportund Werksbeton. Das Produktportfolio umfasst Einzelmaschinen, mobile und stationäre Aufbereitungs- und Betonmischanlagen und den dazugehörigen Service \& Support. Zurzeit beschäftigt SBM an beiden Standorten ca. 165 Mitarbeiter und gehört zur MFL-Gruppe, welche die in Abb. 1 ersichtlichen Tochter- und Schwesterunternehmen unter ein Dach zusammenführt [1].

\section{Motivation aus aufbereitungstechnischer Sicht}

Mobile Aufbereitungsanlagen haben in den vergangenen Jahren einen Siegeszug durch viele Segmente der rohstoffgewinnenden und -verarbeitenden Industrie vollzogen. SBM hat sich durch seine qualitativ hochwertigen mobilen Aufbereitungsanlagen als kompetenter Mitbewerber in einem stetig wachsendenden Marktsegment bewährt. Diese positive Entwicklung soll konsequent fortgesetzt und mit einem mehrjährigen Forschungsprojekt begleitet werden. Zur Unterstützung wurde dazu der Lehrstuhl für Aufbereitung und Veredlung der Montanuniversität Leoben als Forschungspartner beigezogen.

SBM ist sich insbesondere der spezifischen Herausforderungen bewusst, welche ein Betrieb von Mobilanlagen mit sich bringt. Der häufig kampagnenartig geführte Betrieb bedeutet in der Regel, dass die Mobilanlagen mit unterschiedlichen Aufgabenstellungen konfrontiert sind. Beispielhaft seien unterschiedliche Gesteinsarten (hart, mittelhart und weiche Gesteine) und zu erzeugende Körnungsspezifikationen (Korngrößen- und Kornformverteilungen) genannt. Gelingt diese Anpassung nicht, entweder, weil die Mobilanlage dies nicht zulässt, oder weil das Bedienpersonal nicht über die entsprechende Kompetenz verfügt, arbeitet die Mobilanlage u. U. weit außerhalb des optimalen Betriebsfensters. Diese Bedienfehler führen zu erhöhten Betriebskosten, Verlusten an markttauglichen Körnungsprodukten bzw. nehmen Wartungen und Reparatur- bzw. Stillstandszeiten zu.

\section{Kurze Funktionsbeschreibung}

Abb. 2 zeigt einen raupenmobilen Brecher, welcher sich üblicherweise aus den folgenden Hauptkomponenten zusammensetzt:

- Aufgabebunker (1) inklusive Aufgaberinne (7)

- Vorsieb (2), inklusive Bypass (10) und Vorsiebband (9)

- Prallbrecher (3)

- Magnetscheider (5)

- Brecherabzugsband (11) 
Abb. 1: Übersicht der MFLGruppe. (Quelle: SBM Mineral Processing $\mathrm{GmbH}$ )

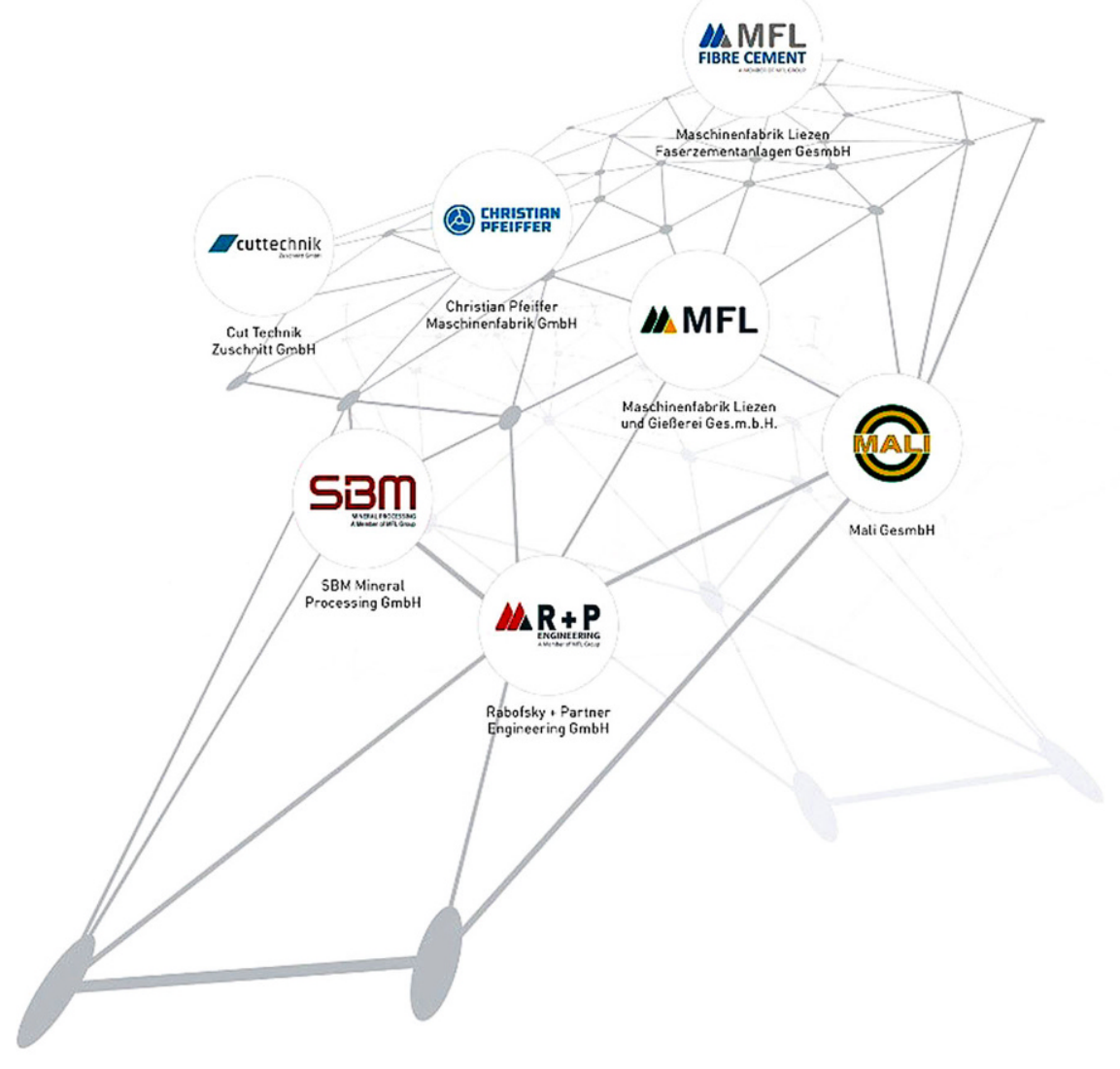

Abb. 2: Hauptkomponenten eines raupenmobilen Prallbrechers. (Quelle: SBM Mineral Processing $\mathrm{GmbH}$ )

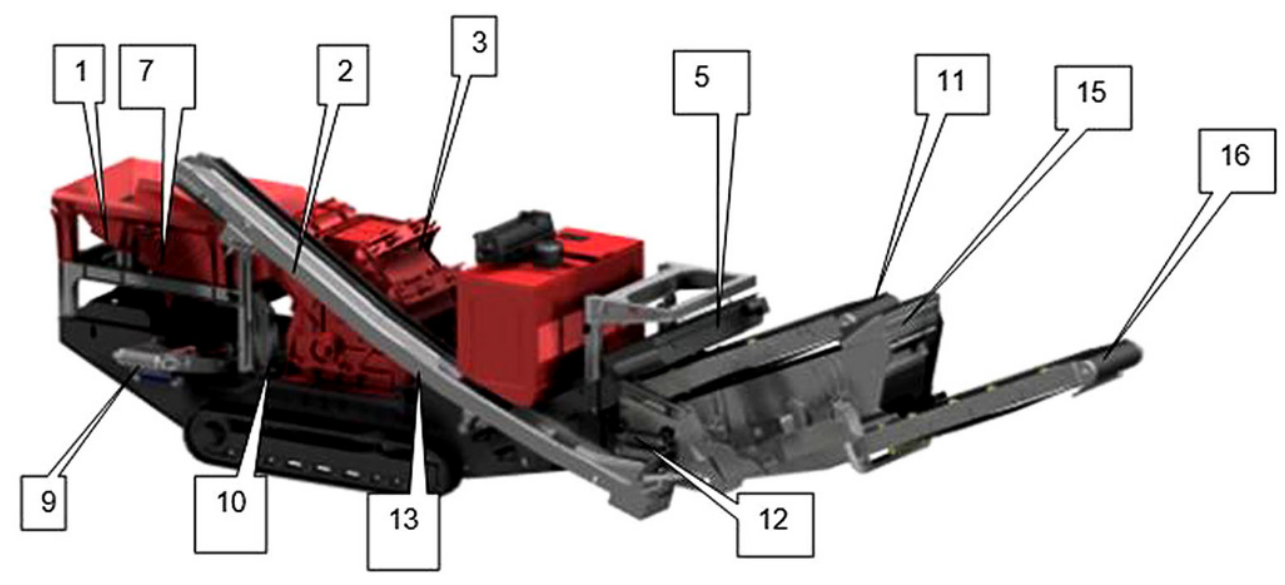

- Klassiersieb (15)

- Übergabe- (12), Rückführ- (13) und Feinkornband (16)

Die Beschickung der mobilen Prallbrecher erfolgt über den Aufgabebunker (1). Sowohl bei SBM als auch den Mitbewerbern werden beim Aufgabebunker ähnliche Systeme mit hydraulisch klappbaren Wänden verwendet. Nach der Beschickung erfolgt die Förderung des Brechgutes in Richtung Brecher mit Hilfe einer Aufgaberinne (7), die über Motoren angetrieben wird. Im Bereich des Vorsiebes (2) erfolgt ein erster Klassierschritt, um den Brecher zu entlasten. Die Klassierung kann ein- oder zweistufig erfolgen oder in manchen Fällen (z. B. bei Asphalt) durch Abdecken nicht benutzt werden. Außerdem kann je nach Kundenwunsch die Feingutfraktion des Vorsiebes mittels des Vorsiebbandes (9) ausgeschleust oder via Bypass (10), am Brecher vorbei, dem Brecherabzugsband (11) aufgegeben werden [2].

Im Prallbrecher (3) wird die Grobgutfraktion des Vorsiebes durch den rotierenden Rotor auf Prallplatten geschleudert und durch Stoßimpulse solange zerkleinert, bis sich ein hinreichender Zerkleinerungseffekt einstellt. Die Einstellung hinsichtlich der zu erreichenden, oberen KorngröBe erfolgt durch den variablen Brechspalt. Einige Hersteller am Markt können den Brechspalt automatisch verstel- 
len, während bei anderen die Verstellung manuell erfolgt. Die automatische Verstellung bringt den großen Vorteil mit sich, dass die Maschine weiter produzieren kann und keine Stillstandzeit anfällt $[3,4]$.

Nach dem Brechvorgang wird das Zerkleinerungsprodukt mittels des Brecherabzugsbandes (11) auf das ein- oder mehrstufige Klassiersieb gefördert und dann klassiert, anschließend über das Feinkornband (16) ausgeschleust bzw. abermalig dem Brecher über das Übergabeband (12) und das Rückführband (13) zugeführt. Direkt nach dem Brecher ist zudem ein Magnetscheider (5) angebracht, um eisenhaltige Begleitmaterialien vom gebrochenen Korn auf einen eigenen Schüttkegel auszuscheiden [2].

Die Energieversorgung erfolgt bei SBM durch eine Motor-Generator-Einheit (diesel-vollelektrisch oder dieselelektrisch), während die meisten Mitbewerber ein dieselhydraulisches Antriebskonzept anwenden. Zur Reduktion von $\mathrm{CO}_{2}$-Emissionen verfügen die meisten der dieselelektrischen Konzepte über die Möglichkeit, die Mobilanlage direkt mit elektrischer Energie aus dem öffentlichen Stromnetz zu versorgen.

\section{Ziele und Entwicklungsinhalte des Forschungsprojektes}

Als forschungsrelevante Ansatzpunkte bei der geplanten Neuentwicklung dienen innovative Werkstoffe, hohe Prozessautomatisierung ( „Iernendes System“) sowie energieoptimierte und $\mathrm{CO}_{2}$-freie Betriebsarten. Bei diesen Ansatzpunkten will SBM die Innovationshebel ansetzen und zum einen die Bedienerfreundlichkeit maßgeblich erhöhen und zum anderen die Mobilanlagen mit der notwendigen Sensortechnik und Software ausstatten, dass diese in die Lage versetzt, unterschiedliche bzw. abweichende Betriebszustände zu erkennen und - angepasst an die vorgegebenen Rahmenbedingungen - selbstständig die richtigen Entscheidungen zur Aufrechterhaltung eines energie- und/ oder produkt-optimierten Betriebes oder zur möglichst raschen Rückkehr in ebendiesen zu treffen. Dabei soll nicht unerwähnt bleiben, dass bereits jetzt ein gewisser Automationsgrad in Mobilanlagen verbaut ist, im Zuge des gegenständlichen Forschungsprojektes soll dieser jedoch drastisch erhöht werden. Dazu ist es notwendig, die vielfältigen Werkzeuge der Digitalisierung auf Einsatztauglichkeit für die Anwendung auf mobile, in einem rauen Betriebsalltag im Einsatz stehenden Aggregate zu prüfen, wozu ein Prototypenbau unerlässlich ist [5].

Im Rahmen eines mehrjährigen, von der österreichischen Forschungsföderungsgesellschaft, kurz FFG, geförderten Forschungsprojekts wurde als übergeordnetes Ziel die Entwicklung eines innovativen, flexibel einsetzbaren, bedienerfreundlichen, sicheren, selbstlernenden, $\mathrm{CO}_{2}$ emissionsfreien und mobilen Prallbrechers definiert.

Darüber hinaus wurden folgende Unterziele definiert:

- Neue Maßstäbe in Richtung autonomes Aufbereiten mit Mobilanlagen durch den Einsatz cyberphysikalischer Systeme und adaptiver Steuerungen zu setzen und damit einen Beitrag zur Al Mission Austria 2030 zu leisten.

- Die Grenzen des Machbaren aus technologischer wie auch wirtschaftlicher Sicht auszuloten bzw. neu zu definieren, die Einsatztauglichkeit mit den Methoden der statistischen Versuchsplanung wissenschaftlich zu untermauern und das Wissen durch eine Schutzstrategie abzusichern.

- Der Rohstoffbranche eine innovative, flexibel einsetzbare und bedienerfreundliche Mobilanlage zur Verfügung zu stellen, die in einer rauen Betriebsumgebung energie- und produkt-optimiert sowie selbstlernend und damit weitestgehend autonom zu arbeiten vermag.

- Das erste $\mathrm{CO}_{2}$-freie Antriebskonzept und umweltschonende/unbedenkliche Bauteilgruppen in der Sparte der Mobilanlagen zu entwickeln und damit ein emissionsfreies Aggregat zu produzieren.

\section{Umweltauswirkungen und Nachhaltigkeit}

Die projektierten Optimierungsmaßnahmen an der Mobilanlage werden zu einer Reduktion des spezifischen Energiebedarfes und damit zu einem geringeren Stromverbrauch bzw. zur Reduktion des $\mathrm{CO}_{2}$-Ausstoßes der Mobilanlage führen. Des Weiteren kann durch den Betrieb am öffentlichen Stromnetz die Mobilanlage auch $\mathrm{CO}_{2}$ neutral betrieben werden (Grünstromvertrag vorausgesetzt). Zusätzlich soll durch die parallel vorangetriebene Entwicklungsarbeit an neuen Antriebkonzepten ein $\mathrm{CO}_{2}$ emissionsfreier Betrieb ermöglicht werden.

Mit den prozesstechnischen Verbesserungen können zukünftig die Lebensdauer der Mobilanlagen erhöht und Maschinenbrüche verhindert werden. Durch weitere Maßnahmen zur Staub- und Schallreduktion sollen die Rahmenbedingungen der Mobilanlage verbessert und der Einsatz der Aggregate im innerstädtischen Bereich gefördert bzw. erst möglich gemacht werden.

Funding. Open access funding provided by Montanuniversität Leoben.

Open Access Dieser Artikel wird unter der Creative Commons Namensnennung 4.0 International Lizenz veröffentlicht, welche die Nutzung, Vervielfältigung, Bearbeitung, Verbreitung und Wiedergabe in jeglichem Medium und Format erlaubt, sofern Sie den/die ursprünglichen Autor(en) und die Quelle ordnungsgemäß nennen, einen Link zur Creative Commons Lizenz beifügen und angeben, ob Änderungen vorgenommen wurden.

Die in diesem Artikel enthaltenen Bilder und sonstiges Drittmaterial unterliegen ebenfalls der genannten Creative Commons Lizenz, sofern sich aus der Abbildungslegende nichts anderes ergibt. Sofern das betreffende Material nicht unter der genannten Creative Commons Lizenz steht und die betreffende Handlung nicht nach gesetzlichen Vorschriften erlaubt ist, ist für die oben aufgeführten Weiterverwendungen des Materials die Einwilligung des jeweiligen Rechteinhabers einzuholen.

Weitere Details zur Lizenz entnehmen Sie bitte der Lizenzinformation auf http://creativecommons.org/licenses/by/4.0/deed.de. 


\section{Literatur}

1. SBM Mineral Processing GmbH Österreich - Über uns, Über SBM, Traditionsunternehmen, 2021, https://www.sbm-mp.at/de/ueberuns.html (25.06.2021)

2. Schwister, K.; Leven, V.: Verfahrenstechnik für Ingenieure - Lehrund Übungsbuch, München: Carl Hanser Verlag GmbH \& Co. KG, 2014.

3. Höffl, K.: Zerkleinerungs- und Klassiermaschinen. Berlin, Heidelberg: Springer, 1986

4. Olshausen, H.-G.: VDI-Lexikon Bauingenieurwesen, Berlin, Heiderlberg: Springer, 1991
5. Jämsä-Jounela, S.-L.: Future Automation Systems in Context of Process Systems and Minerals Engineering. In: IFAC-PapersOnLine, 52 (2019), Nr. 25, S. 403-408, https://doi.org/10.1016/j.ifacol.2019.12. 570 .

Hinweis des Verlags. Der Verlag bleibt in Hinblick auf geografische Zuordnungen und Gebietsbezeichnungen in veröffentlichten Karten und Institutsadressen neutral. 